\title{
Communication skills as a competency in Dental schools: A Review
}

\author{
Vaibhav Karemore
}

Associate Professor, Dept. of Periodontics, Government Dental College and Hospital, Nagpur, Maharashtra, India

* Corresponding Author:

Email: derperiodon@gmail.com

\begin{abstract}
Communication refers to 'the imparting or exchanging of information by speaking, writing or using some other medium'. To ensure good doctor patient relationship, many health profession schools, including Dentistry, has established courses with an emphasis on communication or soft skills. Dental schools are required to ensure whether undergraduates are adequately trained in communication skills, as there is lack of evidence about its competency assessment. Including more newer methods and concepts to inculcate soft skills in dentistry may help to explore better avenues in its training as well as assessment.
\end{abstract}

Keywords: Communication skills, Dentistry.

People based professions needs sound knowledge of communications skills and are equally essential for dentistry. ${ }^{1}$ It is an abutment over which lot of areas of patient care rests such as, first interaction with patient, probing for associated and additional problems, counseling the patient, explaining treatment options, its complications and advising follow-up. ${ }^{2,3}$ These skills are also necessary for explaining risks to the patient, counseling in case of mishap, providing information about a surgical procedure and its complications, taking an informed consent, and lot more other areas of patient care. The empathy that a patient feels by clinician's approach can help to improve their health, mediated through participation in self-care and adherence to treatment. ${ }^{4}$

Curriculum for Dentistry involves dynamic process including domains for clinical, cognitive, and psychomotor skills. ${ }^{1}$ Lecture is the most common teaching method and written examination is more frequently practiced for assessment in dental schools. Though, based on the Miller's pyramid of competence, recently, many methods have been incorporated in the syllabus for better professional competency.

To ensure good doctor patient relationship, as a lifetime goal, many schools has established courses with an emphasis on communication or soft skills. ${ }^{5}$ American Dental Education Association has even recommended evaluation of students' skills for communication, using a competency examination. ${ }^{6}$

It proves that, it is an issue of pivotal need to be addressed at right time. In Indian scenario, communication skills are not formally taught in every dental institute rather students learn them through the clinical teaching by faculty.

Carey $\mathbf{~ J A}$ et $\mathrm{al}^{7}$ stated that, dental schools are required to ensure whether undergraduates are adequately trained in communication skills though there is lack of evidence about its competency assessment. In the contrary, medical institutes are training the undergraduates formally for the same. But, there is tremendous variation among medical schools in the way, and extent to which, communication skills are taught and assessed. $^{8}$

The methods routinely practiced to teach communications skills in majority institutes were didactic learning and clinical role- play using simulated patients. Assessment methods used to evaluate competency in communication skills focused mainly on observer evaluation of student interactions at consultation whereas, patient involvement in training appears to be minimal. It would be interesting to investigate students' perception on the importance of soft skills during their undergraduate dental training and the teachers' perception and their preparedness in teaching and evaluating students' soft skills. ${ }^{1,3,6,9}$

Cannick GF et $\mathrm{al}^{6}$ have used Objective Structured Clinical Examination (OSCE) as a tool to evaluate dental students' competency in interpersonal and tobacco cessation communication skills. All students were evaluated for interpersonal communication skills at baseline and at six months post-OSCE by standardized patients and on their tobacco cessation communication skills by two independent observers. After this one-shot intervention it was concluded that this method of teaching communication skills was not successful. A well planned, comprehensive communication skills training course may be more beneficial than a single, brief training session for improving dental students' communication skills.

Timothy $\mathrm{L}$ et $\mathrm{al}^{10}$ designed a study to observe and document the effect of a course in patient management conducted for third-year dental students at Nova Southeastern University College of Dental Medicine. The course contents were, dealing with anxiety, interviewing techniques, patient records, behavior management, and patient relations. Results were significant and were in favor of the course. This study indicates that effective communication between dental students and their patients may be acquired and refined through a training that includes basic interviewing 
skills. ${ }^{10,11}$

Recently in 2016, Merete Jorgensen and et al ${ }^{12}$ evaluated consultation and communication skills performance of medicine students using rating scales, interview and by a questionnaire which was found to be effective. Author also recommended need of a better tool for exploring the effect of different methods in teaching consultations skills as it is yet to be developed. ${ }^{12,13}$

Anil Choudhary and et $\mathrm{al}^{14}$ in their study design gave training in basic communication and counseling skills to students and taught them patient interviewing technique according. They observed that, significantly higher number of students had a positive attitude toward learning communication skills. Authors also stated that, these results are due to the fact that they are in contact with the patients during clinical posting in final year and begin to understand the importance of effective communication.

Perception of teachers in a dental institute, regarding the importance of applying communication skills learning and assessment methods in dental curriculum was evaluated. $97 \%$ of teachers suggested that a broader approach of communication skill is necessary to be included in the dental curriculum. This study suggests that dental educators must be educated and updated time to time about the recent methods of learning and assessment. ${ }^{1,9,15,16,17}$

Dentistry is known for frequent appointments or sittings for majority of the treatments. Patient complaints regarding dental appointments have commonly been included as communication problems with their dentist. ${ }^{5}$ To deal with such clinical demand, effective communication would aid and hasten the diagnostic process, decision- making and enhance patient-dentist understanding. Therefore inclusion of soft skills in curriculum is necessary in dental profession. . $^{5,18,19}$

Razak IA et $\mathrm{al}^{20}$ assessed the competency of dental graduates from Malaya university. In this evaluation it was found that the five-year university curriculum for better professional competency was well accepted by students. The faculty reduced the number of didactic teaching hours and focused more on self-directed and problem-based learning. Here, author recommends that problem-based learning sessions can improve students' communication skills and reasoning abilities. Dental Graduates in this study believed that their soft skills were developed through undergraduate training.

Many universities have incorporated soft skills training courses for higher education programs. ${ }^{21}$ Students should also be made to realize the importance of sharpening their soft skills so that they can able to compete in the world without hesitations. Communication skills training courses were practiced at university of UM and UKM during five-year dental graduation program. The courses conducted were for five years, divided in sections and short term respectively. It was found that both courses whether long or less extensive, affected students in a positive way. ${ }^{22}$
Several areas of methodology need to be addressed in future studies on communication skills to extend the scope of research to include intra-operative communication and active evaluation by dealing with real patients. ${ }^{7}$

Teachers and students are stakeholders to deal with routine dental practice in the institutional set ups. Teachers' contribution towards professional competency of each student is definitely makes lion's share, so their periodic feedback is necessary. Teachers can be sensitized and trained first for better soft skills teaching programs. The institutes, which are practicing such programs regularly, can be the role models as well as guide for upcoming dental schools.

So far, communications skills were taught and assessed by using innovative methods, such as role play, simulations, small group discussions, videotaped dental interview, real patient, student evaluation questionnaire, behavior role play test, interpersonal communication checklist, videotape reviewing, OSCE, and real patients. All the methods have given promising results, which is proved by positive feedback from students. This suggests for future inclusion of communication skills in dental curriculum and effective implementation of it with refined assessment techniques. Including more newer methods and concepts to inculcate soft skills in dentistry may help to explore better avenues in its training as well as assessment.

Communication refers to 'the imparting or exchanging of information by speaking, writing or using some other medium'. ${ }^{24,25}$ It also includes the mutual conveyance of emotions, knowledge and ideas in an honest and effective manner. Personal qualities and vision would stand as a critical factor for development of soft skills, which further would aid to enhance critical thinking and lifelong learning. Conclusively it can be said that Communication skills is an integral component of Dentistry to achieve excellence with personality development.

\section{References}

1. A Teacher's Perspective on Inclusion of Communication Skills in Dental Education Curriculum. Samer Kasabah, Prashanth Prakash, Syed K Aliuddin. Int J Oral Care Res 2017;5(2):1-6.

2. Tips to enhance effective communication skills for the dental assistant. https://www.dentistryiq.com/.../tips-toenhance-effective-communication-skills-for-the.Jan 22, 2013)

3. Jane Kidd, Vinod Patel, Ed Peile, Yvonne Carter.Clinical and communication skill. BMJ 2005;330:374. Impact of Communication in Healthcare, Institute for Healthcare ...https://healthcarecomm.org/about-us/impact-ofcommunication-in-healthcare/ July 2011)

4. Simone Alvarez. Jobst-Hendrik Schultz. A communication-focused curriculum for dental students an experiential training approach. BMC Medical Education BMC series - open, inclusive and trusted201818:55)

5. Cannick GF, Horowitz AM, Garr DR, et al. Use of OSCE to evaluate brief communication skills training for dental 
students. J Dent Educ 2007:71:1203-09.

6. Carey JA, Madill A, Manogue M. Communications skills in dental education: a systematic research review. Eur $J$ Dent Educ 2010;14(2):69-78.

7. Gregory Makoul. Communication Skills Education in Medical School and Beyond. JAMA 2003;289(1):93. doi:10.1001/jama.289.1.93)

8. Tapasya Karemore. Formal Training of Communication Skills for Dental Undergraduates. Need of an Hour. Elixir Psychol 2017; 112:48940-44.

9. Hottel TL, Hardigan PC. Improvement in interpersonal communication skills of dental students. J Dent Educ 2005:69:281-84.

10. E doherty, C A O'boyle, W Shannon, H Mcgee, G Bury. Communication skills training in undergraduate medicine. Irish Med J 1990;83(2):5412.

11. Kathryn Robertson. Active listening more than just paying attention. Australian Family Physician 2005;34(12).

12. Merete Jorgensen, Klaus Witt. Teaching communications skills to medical students using teaching method and access to online video cases. Med ed publish https://doi.org/10.15694/mep.2016.000116.

13. J Dacre1, J Richardson1, L Noble1, K Stephens, N Parker. Communication skills training in postgraduate medicine: the development of a new course. Postgrad Med J 2004;80:711-15.

14. Anjali Choudhary, Vineeta Gupta.Teaching communications skills to medical students: Introducing the fine art of medical practice. Int J Appl Basic Med Res 2015;5(Suppl 1).

15. Winefield HR, Chur-hansenA. Evaluating the outcome of communication skill teaching for entry-level medical students: does knowledge of empathy increase? Med Educ 2000;34(2):90-4.
16. Maria C Hausberg. Enhancing medical students' communication skills: development and evaluation of an undergraduate training program. BMC Med Educ 2012;12:16

17. Knut Aspergren. Teaching and learning in communication skills in Medicine: A review with quality grading of articles. BEME Guide No. 2.1999:21(6):1-12.

18. Lesley MacDonald-Wicks. Effective teaching of communication to health professional undergraduate and postgraduate students: A Systematic Review. JBI Library of Systematic reviews. 2012;10(28)Suppl.

19. Elizabeth Rider. A model for communication skills assessment across undergraduate curriculum. Med Teacher 2006;28(5):127-34.

20. Razak IA, Latifah RR, Jaafar N, Abu HassanMI, Ab Murat N. Assessing the competency of University of Malaya dental graduates: employers' and graduates' perception. J Dent Educ 2008:72:364-69.

21. Roselina Shakir Soft skills at the Malaysian institutes of higher learning. Asia Pacific Educ Rev 2009;10:309-15.

22. Nor NA, Yusof ZY, Shahidan MN. University of Malaya dental students' attitudes towards communication skills learning: implications for dental education. J Dent Educ 2011;75:1611-19.

23. Noelle Junod Perron. Impact of postgraduate training on communication skills teaching: a controlled study. $B M C$ Med Educ 2014;14:80

24. Gonzalez MA, Abu Kasim NH, Naimie Z. Soft skills and dental education. Eur J Dent Educ 2013;17(2):73-82. doi: 10.1111/eje.12017.

25. Kevin B. Wright Medical Student Attitudes toward Communication Skills Training and Knowledge of Appropriate Provider-Patient Communication: A Comparison of First-Year and Fourth-Year Medical Students. Med Educ Online [serial online] 2006;11:18. 